\title{
HIPPARCOS Data on Pulsating Stars ${ }^{1}$
}

\author{
Catherine Turon \\ Observatoire de Paris-Meudon, DASGAL / CNRS URA 335, \\ 92195 Meudon Cedex
}

Floor van Leeuwen

Royal Greenwich Observatory, Cambridge, CB3 OEZ, UK

\begin{abstract}
The preliminary astrometric and photometric results obtained from the first 30 months of HIPPARCOS data are presented. Examples of the results obtained for the various categories of pulsating variables included in the HIPPARCOS programme are given. The perspectives opened by GAIA (Global Astrometric Interferometer for Astrophysics), the concept for an astrometric interferometer submitted in September 1994 to the ESA Horizon 2000+ survey committee, are briefly exposed.
\end{abstract}

\section{Introduction}

The ESA HIPPARCOS satellite was launched by Ariane in August 1989. Despite the failure of its apogee boost motor, scientific data were obtained from November 1989 to March 1993, i.e., during 37 months. In the course of the data analysis, several intermediate catalogues were constructed by each of the two Consortia (NDAC and FAST) involved in the HIPPARCOS data reduction. The preliminary catalogues obtained from the analysis of the first 30 months of data were merged into a unique 'H30' solution, providing mean values of the positions, parallaxes and proper motions for about 107000 stars, from a total observing programme of 118000 stars. All statistics on astrometric results given below are based on this preliminary 'H30' solution. The photometric results are from the analysis performed by the NDAC Consortium, as the merging between the two Consortia is not yet available.

Comprehensive descriptions of the HIPPARCOS mission, the astrometric data analysis within NDAC and FAST, the photometric aspects, and the contents of the observing programme are described respectively in Perryman et al. (1992), Lindegren et al. (1992), Kovalevsky et al. (1992), Grenon et al. (1992), Turon et al. (1992). The construction of ' $\mathrm{H} 30$ ' and the properties of this intermediate HIPPARCOS catalogue are described by Kovalevsky et al. (1995)

\footnotetext{
${ }^{1}$ Based on observations made with the ESA HIPPARCOS satellite, and on work performed within the INCA, NDAC and FAST Consortia
} 
and Lindegren et al. (1995); the HIPPARCOS photometric database and its application to variability in van Leeuwen et al. (1995).

\section{HIPPARCOS preliminary astrometric results}

Although the final HIPPARCOS catalogue will be more accurate and more complete than this provisional version, $\mathrm{H} 30$ already gives a fair appraisal of the final mission results. For stars with HIPPARCOS magnitudes in the range 8-10 mag, i.e., for the bulk of the observing programme, the mean values for the formal standard errors of the five astrometric parameters in H30 are as follows: 1.2 mas and 1.0 mas for $\alpha \cos \delta$ and $\delta$ respectively; 1.5 mas for the parallax; 1.7 mas/yr and $1.4 \mathrm{mas} / \mathrm{yr}$ for $\mu_{\alpha} \cos \delta$ and $\mu_{\delta}$ respectively (Kovalevsky et al. 1995, Lindegren et al. 1995). These errors vary with the ecliptic latitude (by a factor of about 1.5 from the ecliptic plane to high latitudes), and with the apparent magnitude (by a factor of about 3 from the brightest to the faintest stars).

\section{HIPPARCOS preliminary photometric results}

The photometric data obtained with the HIPPARCOS satellite are in many ways different from data obtained on the ground, primarily because the HIPPARCOS satellite was designed to be an astrometric satellite, and the photometric data form a by-product. Nevertheless, the photometric data obtained are of high quality, and much attention has also been paid to achieve the best possible photometric results. This section gives a brief overview of characteristics of the HIPPARCOS photometry. For more detailed information we refer to Van Leeuwen et al. (1995).

\subsection{Satellite observing mode}

The HIPPARCOS payload was based on what could be called a 'double telescope'. Two regions of the sky, separated by $58^{\circ}$, were observed simultaneously and projected on one focal plane, where there was situated a very precise modulating grid of 0.9 by 0.9 degrees on the sky. The satellite operated in scanning mode, such that the two sky regions seen through the telescope described a great circle on the sky. The rotational period of the satellite was $2^{h} 40^{m}$, causing a star to pass across the grid in 20 seconds. During one rotation of the satellite, the rotation axis of the satellite was moved by approximately 0.4 degrees as a result of its own rotation under an angle of $43^{\circ}$ around the direction to the Sun. This precession of the rotation axis completed 6.4 rotations per year, thus providing a complete scan of the sky in at least two different directions every 6 months.

One effect of this scanning law, as it is usually referred to, is that observations on the ecliptic poles are evenly spaced in time, observations at around ecliptic latitudes of $\pm 47^{\circ}$ come often in clumps, that can last up to 2 days, while observations at lower ecliptic latitude are generally scarce but may also contain one or two clumps. The sky coverage is further complicated due to perigee passages, occultations and van Allen belts crossings, during which no observations were possible. On average, observations were obtained for between 60 and 70 


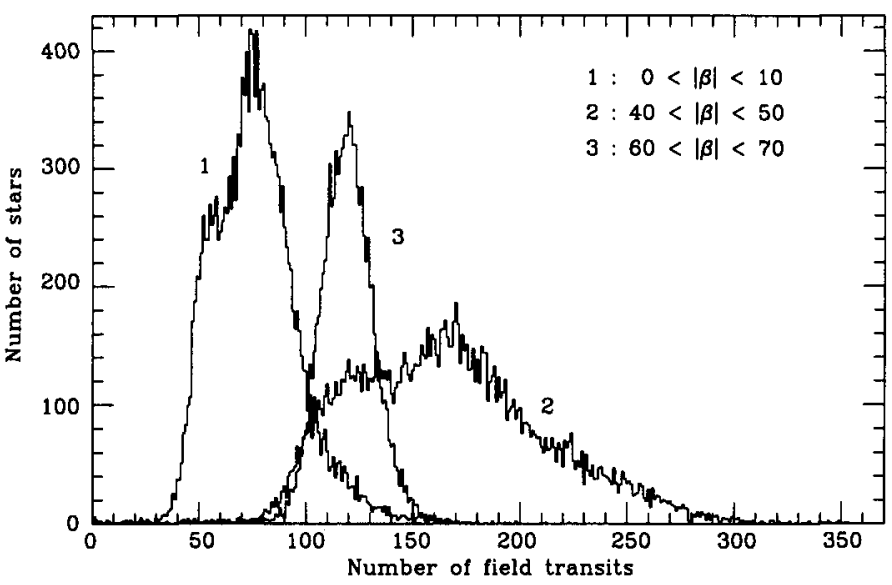

Figure 1. Histograms of the number of observations per star at different ecliptic latitudes

percent of the time. Figure 1 shows histograms of the number of observations per star at different ecliptic latitudes.

A problem unique to the HIPPARCOS photometric data is the superposition of an image from 'the other field of view'. This problem is greatly reduced due to the use of an image dissector tube (IDT from here on). The IDT allows a photomultiplier to observe only a very small region $\left(30^{\prime \prime}\right)$ behind the modulating grid. This still gives the occasional superposition, but these are recognised afterwards and the relevant data is either corrected (for small disturbances) or removed. The IDT also introduced, however, its own complications to the data reductions. The device contains a relatively large number of small lenses allowing it to focus on a small area of the modulating grid. These lenses, when in outer space, get affected by radiation, which changes the transmission as a function of wavelength throughout the mission. This effect could only be calibrated properly when the mission was finished, and the final photometric system has been defined by a passband that represents the situation at the start of 1992 . All data were reduced to that passband as far as possible. A full description of these calibrations and the determination of the passband and its evolution is given by Grenon (1995).

\subsection{Satellite signal}

The photometric data obtained with the HIPPARCOS satellite are derived from the modulated signal recorded by the IDT: we used the zero-level and the amplitude of the first harmonic of the modulation. The zero-level, called the DC component, contains the stronger signal, but for fainter stars it is influenced by a background signal that has not been measured independently. The modulation amplitude (AC component) is free of background influences but is much weaker. In the case of close binaries (separations less than $12^{\prime \prime}$ ) the $\mathrm{AC}$ component is also sensitive to the orientation of the binary system with respect to the modu- 


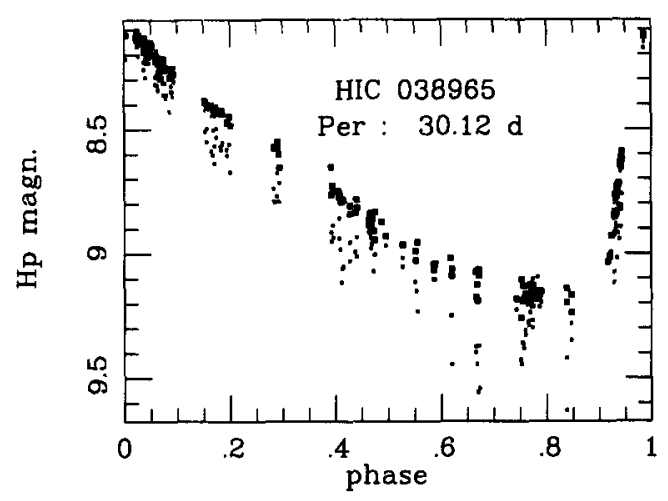

Figure 2. HIPPARCOS observations of the Cepheid AQ Pup; the differences between $\mathrm{DC}$ (filled squares) and $\mathrm{AC}$ (small dots) photometry indicate the presence of a component

lating grid. Thus, by comparing reduced DC and AC based photometric data, it becomes possible to detect duplicity (for more details see Mignard et al, 1995). As an example, Figure 2 shows the reduced HIPPARCOS observations of the Cepheid AQ Pup in both DC (filled squares) and AC (small dots).

The size of the sensitive area of the IDT also means that double stars with separations between $8^{\prime \prime}$ and $20^{\prime \prime}$ are often rather noisy in their DC component: one of the double star's components would be situated near the edge of the aperture where the sensitivity of the IDT reduces sharply.

The IDT samples the light behind the grid at $1200 \mathrm{~Hz}$. Data are collected over a period of 2.133 seconds (2560 sampling intervals). During that time interval each star travels across $11 \%$ of the grid. Within the time interval, between one and 10 , average 4 to 5 , stars are observed almost simultaneously. In the photometric reductions these so-called frame transits are used as input data, allowing the calibration of large scale sensitivity corrections across the modulating grid. In the final output, however, the reduced frame transits belonging to one crossing of the grid for a star are combined to what is called a 'Field Transit'. These field transits form the basis of the photometric data base that will become available alongside the HIPPARCOS astrometric catalogue. During the development and implementation of the photometric reduction process, many tests have been carried out to verify that no undue biases and offsets had developed in the final product. This was further enhanced by the independent reductions done by the two data reduction consortia, which allowed extensive comparisons of reduced data.

In the final HIPPARCOS astrometric catalogue, the main photometric data will be based on the DC component, generally referred to as $H p$. The analysis has involved sophisticated modelling of the background signal. This background signal contains contributions from the diffuse galactic light, the zodiacal light, and the radiation environment (van Allen radiation belts due to the unfortunate elliptical orbit in which the satellite got trapped). Orbits during which 


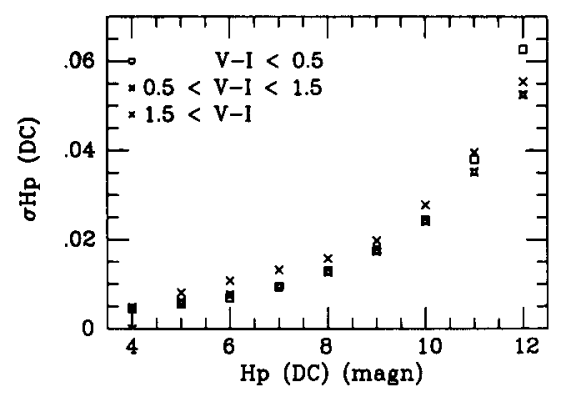

Figure 3. Standard deviations obtained for the individual photometric measurements as a function of magnitude, for three colour ranges

no convergence of the attitude was obtained have been used to calibrate and test the background model. The observed standard deviations obtained for the individual photometric measurements (field transits) are shown in Figure 3 as a function of magnitude for three colour ranges.

Additional photometric information, though of less accuracy and only for stars brighter than 10th magnitude, is provided by the Star Mapper detectors. Here, light is split into a $B_{T}$ and a $V_{T}$ channel, resembling the Johnson system (see Grossmann et al., 1995). The information obtained during the mission from the star mapper photometry has been used to improve the knowledge of colour indices, needed in the reductions of both photometric and astrometric data. Figure 4 shows $\delta$ Cephei as observed in the broad-band $H p$ and $B_{T}-V_{T}$.

\section{HIPPARCOS observing programme}

\subsection{Overall contents}

The HIPPARCOS observing programme has been defined, iteratively, on the basis of 210 scientific proposals submitted to the European Space Agency, while making an optimal use of the observing possibilities of the satellite. It was published as the 'HIPPARCOS Input Catalogue' (HIC, Turon et al. 1992b, 1992c and 1994) including the most up-to-date and homogeneous ground-based information related to the 118000 preselected programme stars. Half of the catalogue consists of a basic list of 'bright' stars, with an apparent limiting magnitude varying with galactic latitude and spectral type. The catalogue is virtually complete for stars brighter than $V=7.9+1.1|\sin b|$ for spectral types earlier than G5 (included), and for stars brighter than $V=7.3+1.1|\sin b|$ for spectral types later than G5. The 60000 fainter stars have been selected from the proposals on grounds of scientific merit.

\subsection{Pulsating variable stars}

Among the proposals, 32 deal with variable stars, of which 21 more specifically deal with pulsating variable stars. The scientific objectives of these proposals relate to many areas of Stellar Physics: determination of intrinsic parameters 


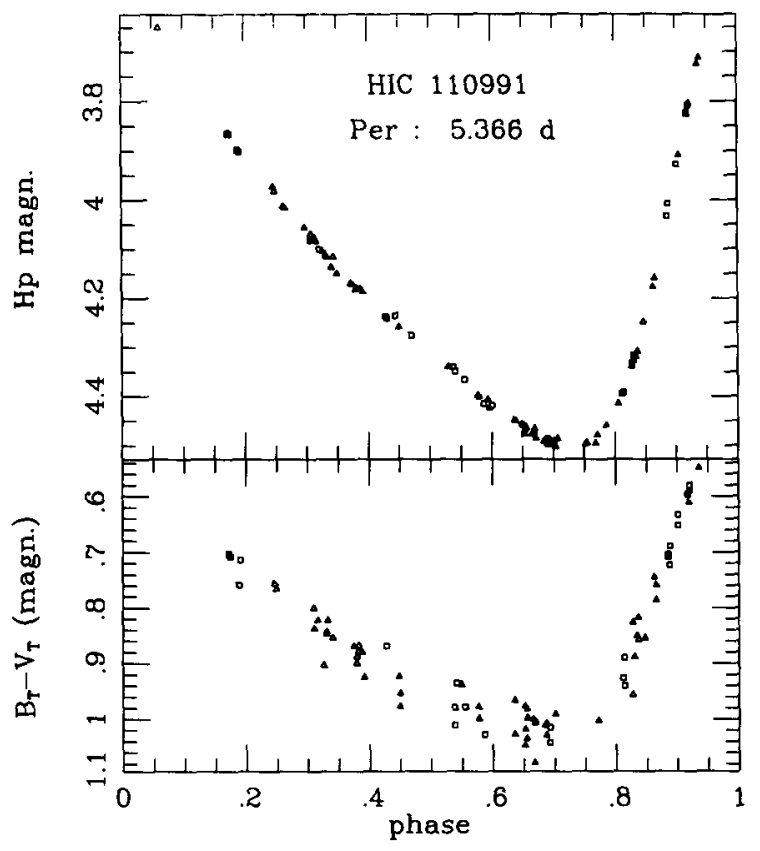

Figure 4. $\delta$ Cephei as observed in the broad-band $H p$ and $B_{T}-V_{T}$

(absolute magnitude, mass, diameter, etc.), internal structure models (understanding of instability strip boundaries), mass transfer, age determination, relations between age, absolute magnitude and metallicity, etc.; Galactic Physics: statistical parallaxes, galactic potential and mass distribution, population and galactic evolution studies (relation of kinematics and metallicity versus age), etc.; Cosmic Distance Scale determination: cluster membership for Cepheids, zeros of period-colour-luminosity relations, kinematics of pulsating variable stars, etc.

5830 known or suspected variable stars are included in the HIPPARCOS observing programme. Among these are 1704 pulsating, 504 eclipsing, 223 rotating, 207 eruptive, 16 cataclysmic and $2 \mathrm{X}$-ray variables. Periods were known for 2031 stars, and amplitudes for 2585 stars. For 244 large amplitude stars, satellite observations made use of ephemerides, prepared in advance in order to obtain correct observing time allocation (Mennessier et al. 1992).

\section{Examples of photometric results on pulsating variable stars}

\section{1. $\delta$ Scuti and Dwarf Cepheids}

The $\delta$ Scuti and dwarf Cepheids are relatively common stars, providing a number of examples well within the HIPPARCOS horizon for parallax measurements. The HIPPARCOS distance calibrations will clarify some of the uncertainties in luminosity for stars like AI Vel and SX Phe. HIPPARCOS cannot contribute much to the discussion of the light variations of these multiple mode variables. 

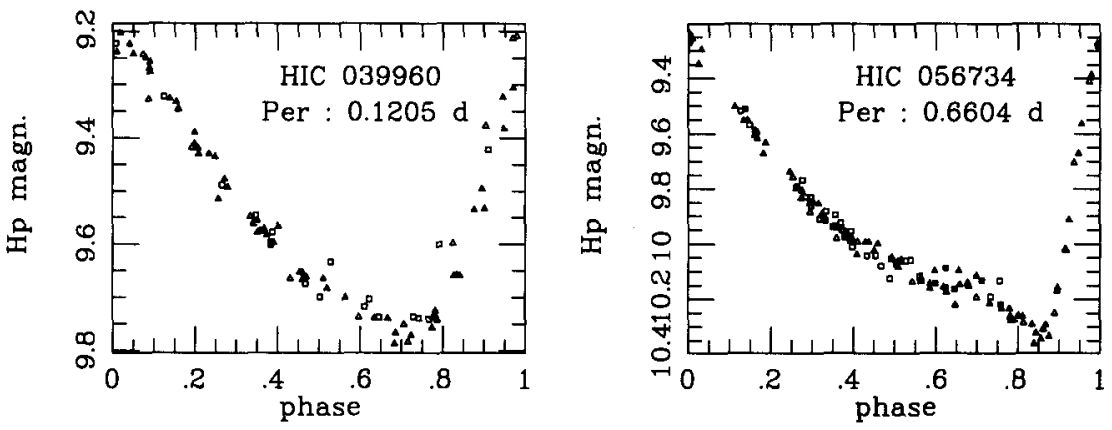

Figure 5. Light curve of the $\delta$ Scuti star SZ Lyn (left), and of the RR Lyrae star SU Dra (right)

For those $\delta$ Scuti stars with a dominant pulsation mode we are, however very well able to establish a pulsation period for the duration of the mission, and compare this with what is available in the literature. Figure 5 (left) shows the data for one of the few single period $\delta$ Scuti stars in our sample: SZ Lyn (HIC 39960).

\subsection{RR Lyrae stars}

There are only a few RR Lyrae stars close enough to obtain a marginal parallax measurement with HIPPARCOS. The HIPPARCOS photometry worked out very well for RR Lyraes. The usually single, short periods received good random coverage and a good chance to obtain accurate periods over the mission time span. In combination with data from the literature this allows a systematic study for period changes. Figure 5 (right) shows an example of HIPPARCOS measurements for the star SU Dra (HIC 56734). Among the original selection of RR Lyrae stars in the HIPPARCOS Input Catalogue there are at least 8 misidentifications (too bad a priori coordinates) and two suspected RR Lyrae stars that turned out to be more likely $\beta$ Lyr or W UMa stars (IV Pav and BX Dra).

\section{3. $\delta$ Cephei stars}

Like the RR Lyrae stars, there are only very few Cepheids with marginal distance determinations in the HIPPARCOS catalogue. These lie generally in the expected range. The photometric coverage of the short period Cepheids is generally good, but it deteriorates towards longer periods, when observations become rather clumped together and coverage is usually poor. For double mode Cepheids like TU Cas, the coverage is too scarce to derive reliable information on its various frequencies. One new Cepheid, HIC 23768, was discovered among the HIPPARCOS stars. Figure 6 (left) shows the accumulated data for this star (spectral type G5), folded with a period of $\mathbf{3 . 2 9 4 8 3}$ days. 

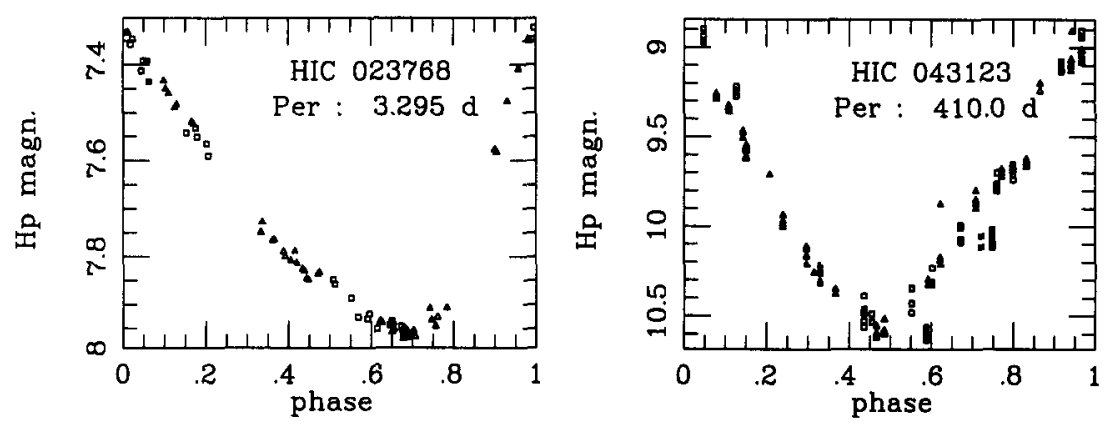

Figure 6. Light curve of a new Cepheid (left), and of a new semi regular- red giant (right)

\subsection{Irregular $\mathrm{K}$-giants and Miras}

The photometric coverage of the long period semi regular and irregular variables is in general poor. In many cases the presentation of the HIPPARCOS photometry will be supported by calibrated data obtained by the AAVSO, to show the HIPPARCOS data points in a wider context. Where the HIPPARCOS data may well be useful is in the study of secondary short term variations, which are occasionally well covered (for up to 2 days) and of an accuracy much better than can be achieved with the AAVSO data. Many new semi regular and irregular K and $M$ giants have already been found in the HIPPARCOS photometric data. Figure 6 (right) shows an example of one such discovery (HIC 43123).

\section{Astrometric results on pulsating variable stars}

The preliminary results obtained in the $\mathrm{H} 30$ solution for the trigonometric parallaxes of all types of pulsating variable stars are given in Table 1 . The astrometric data for $\delta$ Scuti, $\beta$ Cephei, slow irregular, and semiregular variables will be especially improved. In addition, distance determinations will be obtained for two SX Phœnicis and one ZZ Ceti variables. An HR diagram drawn for $\delta$ Scuti stars using astrometric and photometric data from the satellite, immediately shows some misclassifications among these stars. Good accuracies will also be obtained for a few RR Lyræ and Cepheids. The periods of the Cepheids with relative precisions of the $\mathrm{H} 30$ parallax better than $30 \%$ range from 1.9 to 9.8 days. Final results will slightly improve these statistics.

Besides these direct improvements, indirect important improvements will come from a new calibration of photometric and spectroscopic distances of spectral types $\mathrm{F}$ and $\mathrm{G}$, from an excellent direct distance determination of about 20 open clusters of various metallicities, from a much more reliable calibration of the HR diagram main sequence versus metal and helium contents, from a much 
Table 1. Pulsating variables in H30. Relative precisions on parallax

\begin{tabular}{|c|c|c|c|c|c|c|c|}
\hline Type & $\begin{array}{l}\text { Observed } \\
\text { stars }\end{array}$ & $\begin{array}{l}\text { Stars } \\
\text { in } \mathbf{H 3 0}\end{array}$ & $\begin{array}{l}\text { Stars in } \\
\text { clusters }\end{array}$ & $<0.05$ & $\begin{array}{c}\text { (sigma } \pi \\
0.05-0.10\end{array}$ & $\begin{array}{l}\pi) \text { in } \mathrm{H} 30 \\
0.10-0.20\end{array}$ & $0.20-0.30$ \\
\hline $\begin{array}{l}\alpha \text { Cyg } \\
\beta \text { Cep } \\
\text { Cep } \\
\text { CW } \\
\delta \text { Cep }\end{array}$ & $\begin{array}{r}21 \\
68 \\
30 \\
30 \\
250\end{array}$ & $\begin{array}{r}19 \\
61 \\
25 \\
25 \\
235\end{array}$ & $\begin{array}{r}2 \\
10 \\
0 \\
0 \\
6\end{array}$ & $\begin{array}{l}0 \\
0 \\
0 \\
0 \\
0\end{array}$ & $\begin{array}{l}0 \\
3 \\
1 \\
0 \\
1\end{array}$ & $\begin{array}{r}0 \\
16 \\
2 \\
0 \\
2\end{array}$ & $\begin{array}{l}0 \\
9 \\
0 \\
0 \\
2\end{array}$ \\
\hline $\begin{array}{l}\delta \text { Sct } \\
\text { L } \\
\text { M } \\
\text { PV Tel } \\
\text { RR }\end{array}$ & $\begin{array}{r}140 \\
290 \\
239 \\
3 \\
180\end{array}$ & $\begin{array}{r}126 \\
278 \\
178 \\
3 \\
151\end{array}$ & $\begin{array}{r}14 \\
7 \\
0 \\
0 \\
0\end{array}$ & $\begin{array}{r}15 \\
2 \\
0 \\
0 \\
0\end{array}$ & $\begin{array}{r}43 \\
10 \\
0 \\
0 \\
0\end{array}$ & $\begin{array}{r}30 \\
36 \\
4 \\
0 \\
0\end{array}$ & $\begin{array}{r}15 \\
54 \\
5 \\
3 \\
3\end{array}$ \\
\hline $\begin{array}{l}\text { RV } \\
\text { SR } \\
\text { SXP } \\
\text { ZZ }\end{array}$ & $\begin{array}{r}11 \\
435 \\
6 \\
1\end{array}$ & $\begin{array}{r}10 \\
399 \\
6 \\
1\end{array}$ & $\begin{array}{l}0 \\
8 \\
0 \\
0\end{array}$ & $\begin{array}{l}0 \\
0 \\
0 \\
1\end{array}$ & $\begin{array}{l}0 \\
7 \\
1 \\
0\end{array}$ & $\begin{array}{r}0 \\
29 \\
1 \\
0\end{array}$ & $\begin{array}{r}0 \\
51 \\
0 \\
0\end{array}$ \\
\hline
\end{tabular}

more convincing definition of the variable star's cluster membership, and from the discovery of many new periodic variable stars.

\section{Perspectives opened by GAIA}

One of the likely cornerstone missions planned by ESA for the period 2010 to 2020 is a follow up for HIPPARCOS, capable of measuring parallaxes to accuracies of 0.02 mas down to 15 th magnitude, and 3 to 5 times more accurate for brighter stars. The mission could do a complete survey of the sky down to that magnitude, and would measure absolute distances to several globular clusters (47 Tuc, $\omega$ Cen, M3, M5, M15, etc.), tens of open clusters and all observable galactic pulsating stars. A possible concept for such a mission, GAIA (Lindegren \& Perryman, 1994), includes 6 channel (intermediate bandwidth) photometry (accuracies down to 12th magnitude better than 1 milli-mag, and a. few hundredth of a magnitude at 16th mag.) and an additional survey with reduced (1 mas accuracy) positional accuracy for all objects down to 19th or 20th magnitude. GAIA is supported by the extensive experience obtained with the HIPPARCOS mission.

The proposed mission length is 5 years (minimum), which provides an average of 200 to 300 observations per star for each of the 50 million target stars. It will, for example, be able to detect any RR Lyrae star up to a distance of about $7 \mathrm{kpc}$, (expect a few thousand) and assign a meaningful distance to all; and to provide a good distance determination of the brightest Cepheids in the Magellanic Clouds. Using the multi-colour photometry, reddening corrections can be determined as well as surface gravity values, effective temperatures and metallicities.

This mission would be capable of establishing the influence of metallicity on absolute magnitudes, the relation to age of various forms of pulsation and in 
general finally provide the solid foundation that is needed for the developments in astronomy and astrophysics that can be expected for the next century.

\section{Conclusion}

The contribution of HIPPARCOS to pulsating variable star studies is multifold: homogeneous photometric observations; the expected discovery of many new periodic variables (already found up to now, one Cepheid, twenty eclipsing binaries, many semi-regular and irregular red giants), and thousands of microvariables; direct high accuracy distance determination for a small number of stars of almost each type of variable; much more reliable calibrations of photometric and spectroscopic distances, of magnitude versus metallicity and helium contents relations can also be expected.

Acknowledgments. We are grateful to D. Morin, M.J. Penston, D.W. Evans, F. Mignard and M. Frœschlé for their help during the preparation of this paper.

\section{References}

Grenon, M., Mermilliod, M., \& Mermilliod, J.C. 1992, A\&A, 258, 88

Grenon, M., et al. 1995, A\&A,

Grossmann, et al. 1995, A\&A,

Kovalevsky, J., Falin, J.L., Pieplu, J.L., Bernacca, P.L., Donati, F., Frœschlé, M., Galligani, I., Mignard, F., Morando, B., Perryman, M.A.C., Schrijver, H., van Daalen, D.T., van der Marel, H., Villenave, M., Walter, H.G., et al. 1992, A\&A, 258, 7

Kovalevsky, J., Lindegren, L., Froeschlé, M., van Leeuwen, F., Perryman, M.A.C., Falin, J.L., Mignard, F., Penston, M.J., Petersen, C., Donati, F., Hering, R., Høg, E., van der Marel, H., Schrijver, H., \& Walter, H.G. 1995, A\&A, Lindegren, L., Høg, E., van Leeuwen, F., Murray, C.A., Evans, D.W., Penston, M.J., Perryman, M.A.C., Petersen, C., Ramamani, N., Snijders, M.A.J., Söderhjelm, S., Andreasen, G.K., Cruise, A.M., Elton, N., Lund, N., \& Poder, K. 1992, A\&A, 258, 18

Lindegren, L. \& Perryman, M.A.C., September 1994

Lindegren, L., Röser, S., Schrijver, H., Perryman, M.A.C., van Leeuwen, F., Mignard, F., Falin, J.L., Lenhardt, H., Kovalevsky, J., Froeschlé, M., Bernacca, P.L., \& Lattanzi, M. 1995, A\&A,

Mennessier, M.O., Barthès, D., Boughaleb, H., Fighueras, F., \& Mattei, J.A. 1992, A\&A, 258, 99

Mignard, F. et al. 1995, A\&A,

Perryman, M.A.C., Høg, E., Kovalevsky, J., Lindegren, L., Turon, C., Bernacca, P.L., Crézé, M., Donati, F., Grenon, M., Grewing, M., van Leeuwen, F., van der Marel, H., Murray, C.A., Le Poole, R., \& Schrijver, H. 1992, A\&A, 258, 1

Turon, C., Gómez, A., Crifo, F., Crézé, M., Perryman, M.A.C., Morin, D., Arenou, F., Nicolet, B., Chareton, M., \& Egret, D. 1992a, A\&A, 258, 74

Turon, C., et al. 1992b, ESA SP-1136 
Turon, C., et al. 1992c, Bull. Inf. CDS, 41, 9

Turon, C., Morin, D., Arenou, F., Perryman, M.A.C., \& Priou, D., 1994, CDROM version of the HIPPARCOS Input Catalogue, Turon C. ed.

van Leeuwen, $F$., et al. 1995, in preparation

\section{Discussion}

Nemec: I do not see SX Phe in your $M_{V}, \mathrm{~B}-\mathrm{V}$ diagram at $M_{V}=+3.04,(\mathrm{~B}-\mathrm{V})$ $=0.24$ (Nemec, Nemec \& Lutz 1994 AJ 108,222). [Laughter] Is your diagram censored or are our models so much in error?

Turon: The diagram is not censored! Answer to your question about your models in 1996!

Bradley: You do have a ZZ Ceti star in your list? I assume the star is G226-29, the closest and brightest one. I predict the parallax of G226-29 is 95.5 mas.

Turon: Yes, there is a ZZ Ceti star in the HIPPARCOS observing programme. Answer to your prediction in 1996 ! 